Z. Klin. Chem. Klin. Biochem.

13. Jg. 1975 , S. $133-135$

\title{
Spektrophotometrische Bestimmungsmethode für Serumcholinesterase(EC 3.1.1.8)-Varianten mit Succinylbischolin als Substrat
}

\author{
Von D. P. Agarwal, S. Schwenkenbecher, L. M. Srivastava und H. W. Goedde \\ Aus dem Institut für Humangenetik (Direktor Prof. Dr. H. W. Goedde) der Universität Hamburg
}

(Eingegangen am 4. April 1974/10. Februar 1975)

Es wird eine einfache und schnelle Methode zur Bestimmung der Hydrolyse von Succinylbischolin durch Serumcholinesterase (EC 3.1.1.8)-Varianten beschrieben. Succinylbischolin als Substrat hat gegenüber anderen Substraten (Acetylcholin, Benzoylcholin und Butyrylcholin), die klinisch keine Bedeutung haben, verschiedene Vorteile.

Cholin, das Hydrolyseprodukt von Succinylbischolin, wird durch Cholinoxidase (EC 1.1.99.1) zu Betainaldehyd oxidiert. Die Reaktion der Cholinoxidase aus Rattenlebermitochondrien wird gekoppelt mit der Reduktion von Cytochrom c und wird bei $550 \mathrm{~nm}$ gemessen. 50 normale Seren (Phänotyp UU) 17 heterozygote (UA) und 8 atypische (AA) wurden mit dieser Methode getestet. Durch Hemmung der Enzymreaktion der Succinylbischolinumsetzung mit Dibucain (Cinchocain; Kalow, W. \& Genest, K. (1957) Canad. J. Biochem. Physiol. 35, 339-346) wurden 3 Gruppen erhalten. Die Ergebnisse sind vergleichbar mit denen der Standardmethode nach Kalow \& Genest (s. o.), in der Benzoylcholin als Substrat benutzt wird.

A spectrophotometric method for the determination of serum cholinesterase variants with succinyl choline as substrate

A simple and rapid method for the estimation of the hydrolysis of succinyl choline by serum cholinesterase variants is described. Succinyl choline, as substrate for the enzyme assay, has many advantages over other substrates (acetyl choline, benzoyl choline and butryl choline) which have no clinical application. Choline, the hydrolytic product of succinyl choline, is oxidized to betaine aldehyde by choline oxidase (EC 1.1.99.1), a rat liver mitochondrial preparation; this is coupled to the reduction of cy tochrome $c$ which is measured at $550 \mathrm{~nm}$. Fifty normal sera (UU), 17 heterozygous (UA) and 8 atypical (AA) were tested with this method, and on the basis of resistance to dibucaine (Cinchocain; Kalow, W. \& Genest, K. (1957) Canad. J. Biochem. Physiol. 35, 339-346) inhibition, three distinct groups could be established using succinyl choline as substrate. These results are comparable with the standard optical method of Kalow \& Genest (cf. above) using benzoyl choline as substrate.

Das Muskelrelaxans Succinylbischolin (Suxamethonium) wird von der Serumcholinesterase über Succinylmonocholin zu Succinat und Cholin abgebaut. Bei Fehlen der Cholinesteraseaktivität oder bei Vorliegen bestimmter erblicher Enzymvarianten tritt nach Verabreichung des in der Anästhesie häufig verwendeten Muskelrelaxans eine stark verlängerte Apnoe auf.

Durch Anwendung des Hemmtests nach Kalow \& Genest (1) mit dem Lokalanästhetikum Dibucain (Cinchocain) als Inhibitor sowie einiger anderer vereinfachter Methoden (2-9) kann die Existenz von Cholinesterase-Varianten nachgewiesen werden. Bei der wohl exaktesten Methode nach Kalow (1) wird Benzoylcholin als Substrat eingesetzt, das spezifisch durch die Serumcholinesterase einen sehr schnellen Abbau erfährt und spektrophotometrisch direkt bei $240 \mathrm{~nm}$ gemessen werden kann. In diesem Test hemmt Dibucain das normale Enzym zu $>70 \%$. Die atypischen Varianten lassen sich in eine Gruppe von Heterozygoten (DN = Dibucainzahl von 40-70\%) und eine Gruppe von Homozygoten ( $\mathrm{DN}<40 \%$ ) unterteilen. Diese Methode hat allerdings den Nachteil, daß hier das Substrat Benzoylcholin in den Test eingesetzt wird. Da mit Benzoylcholin jedoch nur bei etwa $70 \%$ der in der
Klinik beobachteten Apnoefällen genetisch bedingte Varianten nachweisbar sind (Goedde et al. (10)), könnte eine Untersuchung mit Succinylbischolin als Substrat hier eine weitere Klärung bringen. Die bisher bekannten Methoden zur Messung der Hydrolyse des Succinylbischolin selbst sind jedoch wesentlich aufwendiger wie z. B. der manometrische Test (11), die indirekte Bestimmung unter Verwendung eines Antiserums gegen Cholinesterase (12), der Test unter Verwendung radioaktiv markierten Succinylbischolins (13), die Bestimmung am isolierten Froschmuskel (14), Messung der pH-Änderung (15) u. a.

\section{Methodik}

Prinzip

Die hier beschriebene Methode basiert auf folgender Reaktion:

Succinylbischolin $\stackrel{\text { Cholinesterase }}{\longrightarrow}$ Cholin + Succinat

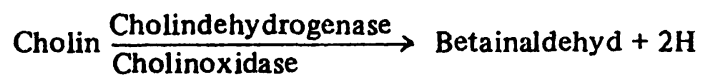

Cytochrom $\mathrm{c}+2 \mathrm{H} \stackrel{\text { Cytochrom c-Oxidasc }}{\longrightarrow}$ reduz. Cytochrom $\mathrm{c}$ 
Das reduzierte Cytochrom c wird in Gegenwart von KCN (um cine Reoxidation zu verhindern) bei $550 \mathrm{~nm}$ im Spektrophotometer gemessen. Rattenlebermitochondrien-Präparation wird als Quelle für Cholinoxidase und Cytochrom c-Oxidase benutzt.

\section{Reagenzien und Lösungen}

Alle Reagenzien wurden in Pufferlösung hergestellt. Trismaleinatpuffer, $0,1 \mathrm{~mol} / 1$ (enthaltend $0,5 \mathrm{mmol} / 1 \mathrm{NaCl}$ und $0,16 \mathrm{mmol} / 1 \mathrm{MgCl}$ ): $\mathrm{pH} 7,0$ (Merck/Darmstadt) Cytochrom c, $57,5 \mathrm{~mol} / 1$ (Boehringer/Mannheim) Rattenlebermitochondrien-Präparation (frisch hergestellt, s. unten)

Testserum (unverdünnt einsetzen)

Succinylbischolin-Chlorid, 9,6 mmol/1 (Sigma/USA)

Benzoylcholin-Chlorid, 9,6 mmol/1 (Fluka/Schweiz)

Dibucain (Cinchocain-Chlorid), 1,92 mmol/1 (Fluka/Schweiz)

\section{Testansatz}

\section{Ungehemmte Reaktion:}

Puffer

$1200 \mu 1$

Cytochrom c-Lösung

$\mathrm{KCN}$-Lösung

Rattenlebermitochondriensuspension

$1000 \mu 1$

$200 \mu 1$

$200 \mu 1$

Serum

$200 \mu 1$

Substrat (Succinylbischolin oder Benzoylcholin) $\quad 200 \mu \mathrm{l}$

Inkubationsvolumen $3000 \mu \mathrm{l} ; 5$ min Vorinkubation; registrierende Messung (Linearität etwa $15 \mathrm{~min}$ ) bei $25^{\circ} \mathrm{C}$ und $550 \mathrm{~nm}$ gegen Leerwert (Puffer).

\section{Gehemmte Reaktion}

Zur Errechnung der Dibucainzahl enthält ein zweiter Ansatz zusätzlich $200 \mu$ l Dibucain-Lösung und entsprechend nur $1000 \mu 1$ Puffer. Alle anderen Reagenzien bleiben unverändert. Die Reaktion wird mit Substrat gestartet.

Berechnung der Aktivität $(\Delta E)$ und des Hemmwertes (DN $=$ Dibucainzahl)

$\Delta E=$ Die Serumaktivität ergibt sich aus der Extinktionsdifferenz über $3 \mathrm{~min}$ mit und ohne Zusatz von Dibucain

$\mathrm{DN}=$

$=100 \cdot\left(\frac{\Delta \mathrm{E} \text { ungehemmte Reaktion }-\Delta \mathrm{E} \text { gehemmte Reaktion }}{\Delta \mathrm{E} \text { ungehemmte Reaktion }}\right)$

Mesșung der Aktivität und Hemmkonstanten nach Kalow und Genest (1)

Zur Differenzierung der Enzymvarianten wird im spektrophotometrischen Test mit Benzoylcholin als Substrat die Relation der Reaktionen mit und ohne Zusatz des Inhibitors Dibucain in Quarzküvetten ( $10 \mathrm{~mm}$ Lichtweg) bei $240 \mathrm{~nm}$ bestimmt (s. Goedde et al., 1967 (12)).

Präparation von Rattenlebermitochondrien (Cholinoxidase $(16,17)$ )

Die Ratten werden mit Äther getötet, ihre Lebern entnommen, in physiologischer $\mathrm{NaCl}$-Lösung gespült, zwischen Fließpapier gepreßt und weitgehend getrocknet. Je $1 \mathrm{~g}$ Leber wird mit $10 \mathrm{ml} 0,25 \mathrm{~mol} / 1$ Saccharose mit einem Homogenisator (nach Potter \& Elvejhem) bei $1500 \mathrm{U} / \mathrm{min}$ homogenisiert. Die Suspension wird durch ein Leinentuch filtriert und $10 \mathrm{~min}$ mit einer Beschleunigung von $700 \mathrm{~g}$ zentrifugiert. Der Überstand wird 20 min mit $24000 \mathrm{~g}$ zentrifugiert. Nun wird der Überstand

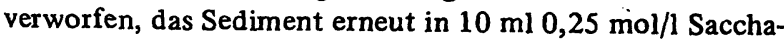
rose homogenisiert und das Homogenat mit $24000 \mathrm{~g} 20 \mathrm{~min}$ zentrifugiert. Der Vorgang wird wiederholt; zum Schluß wird das Sediment in $3 \mathrm{ml} \mathrm{0,25} \mathrm{mol} / 1$ Saccharose homogenisiert und als Suspension verwendet. Das Mitochondrienpräparat zeigt bei Aufbewahrung in Eiswasser über $3 \mathrm{~h}$ keinen Aktivitätsverlust; für 3 bis 4 Wochen kann es in kleinen Teilmengen bei . $-20^{\circ} \mathrm{C}$ mit geringem Aktivitätsverlust gelagert werden.

\section{Ergebnisse und Diskussion}

75 Seren ( 50 UU, 17 UA und 8 AA) wurden mit der neuen Methode sowohl mit Succinylbischolin als auch mit Benzoylcholin als Substrat untersucht. Die Phänotypen der verschiedenen Proben wurden vorher mit Hilfe der Standardmethode nach Kalow \& Genest (1) mit Benzoylcholin als Substrat bestimmt. Die Ergebnisse sind in Tabelle 1 zusammengefaßt; es handelt sich jeweils um Doppelbestimmungen. Die Werte wurden als reproduzierbar angesehen, wenn Reihenuntersuchungen mit 3 unausgewählten Seren durchgeführt wurden (Auswahl der Analyssen: 12; VK = 5,6\%). Die an verschiedenen Tagen durchgeführten Untersuchungen der Esterase-Aktivität șowie auch der Hemmung mit Dibucain zeigten einen Variations-Koeffizienten von etwa $6 \%$. Diese Unterschiede sind jedoch vertretbar, weil dadurch keine Verschiebungen aus der Gruppe bestimmter Phänotypen (Varianten) in eine andere Gruppe bei Bestimmung der DN beobachtet werden.

Wie aus den Ergebnissen ersichtlich, wird bei Verwendung von Benzoylcholin als Substrat keine signifikante Veränderung der DN bei normalen und atypischen Seren gefunden; bei Verwendung von Succinylbischolin als Substrat wird jedoch eine Verschiebung der DN in allen drei Phänotypen beobachtet.

Normale Seren (UU) zeigten eine durchschnittliche DN von 53 gegenüber einer DN von 77 mit.Benzoylcholin; UA und AA hatten eine DN von 25 und 4, gegenüber 58 und 24. Aufgrund dieser Ergebnisse lassen sich die verschiedenen Phänotypen unter Verwendung von Succinylbischolin in drei verschiedene Klassen einteilen: Über DN 40 für Normale (UU), DN 20-40 für Heterozygote (UA) und unter 20 für den atypischen Phänotyp (AA).

Tab. 1. Eingruppierung der Serumcholinesterase-Varianten durch Hemmung mit Dibucain (Prozentuale Hemmung). Die in Klammern gegebenen Werte zeigen den Streubereich der Dibucainzahl für die verschiedenen Phänotypen.

\begin{tabular}{|c|c|c|c|c|}
\hline \multirow[t]{4}{*}{ Phänotypen } & \multirow[t]{4}{*}{$\begin{array}{l}\text { Seren } \\
\mathrm{n}\end{array}$} & \multicolumn{3}{|c|}{$\begin{array}{l}\text { Dibucainzahl (DN), } \\
\text { Mittelwerte } \pm \text { Standardabweichung }\end{array}$} \\
\hline & & Testsystem & & \\
\hline & & $\begin{array}{l}\text { Nach Kalow } \\
\text { \& Genest (1) } \\
\text { mit } \\
\text { Substrat: }\end{array}$ & \multicolumn{2}{|c|}{ Diese Arbeit, mit Substrat: } \\
\hline & & $\begin{array}{l}\text { Benzoyl- } \\
\text { cholin }\end{array}$ & $\begin{array}{l}\text { Benzoyl- } \\
\text { cholin }\end{array}$ & $\begin{array}{l}\text { Succinylbis- } \\
\text { cholin }\end{array}$ \\
\hline $\begin{array}{l}\text { Normal } \\
\text { (UU) }\end{array}$ & 50 & $\begin{array}{l}76,9 \pm 5,8 \\
(>70)\end{array}$ & $\begin{array}{l}76,4 \pm 8,3 \\
(>65)\end{array}$ & $\begin{array}{l}52,9 \pm 11,9 \\
(>40)\end{array}$ \\
\hline $\begin{array}{l}\text { Heterozygot } \\
\text { (UA) }\end{array}$ & 17 & $\begin{array}{l}58,4 \pm 10,7 \\
(40-70)\end{array}$ & $\begin{array}{l}54,2 \pm 7,3 \\
(45-65)\end{array}$ & $\begin{array}{l}25,0 \pm 9,6 \\
(20-40)\end{array}$ \\
\hline $\begin{array}{l}\text { Atypisch } \\
\text { (AA) }\end{array}$ & 8 & $\begin{array}{l}24,1 \pm 14,5 \\
(<40)\end{array}$ & $\begin{array}{l}33,0 \pm 7,0 \\
(<40)\end{array}$ & $\begin{array}{l}3,9 \pm 5,2 \\
(<20)\end{array}$ \\
\hline
\end{tabular}


Trotz der kleinen Anzahl von atypischen Seren ist auffällig, daß die normalen Phänotypen der Cholinesterase bei der Verwendung von Succinylbischolin als Substrat anstelle von Benzoylcholin im Hemmtest mit Dibucain voneinander und von den normalen Phänotypen unterschieden werden können.

Bei allen Personen, bei denen im Hemmtest nach Kalow das ,dibucainresistente“ Enzym festgestellt wurde, wurde auch nach Gabe von Suxamethonium eine verlängerte Apnoe beobachtet. Andererseits ließ sich, wie oben erwähnt, bei Untersuchungen von Apnoefällen nur in etwa $70 \%$ das atypische Enzym nachweisen (10, 19-23). Mit diesem relativ einfach durchzuführenden Test lassen sich vielleicht bei den verbleibenden $30 \%$ der ungeklärten Apnoefälle weitere Enzym-Varianten

\section{Literatur}

1. Kalow, W. \& Genest, K. (1957), Can. J. Biochem. Physiol. $35,339-346$.

2. Harris, H. \& Whittaker, M. (1961), Nature (London) 191 , 496-498.

3. Harris, H. \& Robson, E. B. (1963), Lancet II, 218-221.

4. Morrow, A. C. \& Motulsky, A. G. (1968), J. Lab. Clin. Med. $71,350-356$.

5. Swift, M. R. \& La Du, B. N. (1966), Lancet $I, 513-514$.

6. Ashby, T. M., Suggs, J. E. \& Jul, D. L. (1970), Clin. Chem. $16,503-506$.

7. Goedde, H. W. \& Fuss, W. (1964), Klin. Wochenschr. 42, 286-289.

8. Garry, Ph. (1971), Clin. Chem. 17, 192-198.

9. Smith, J. C. \& Foldes, F. F. (1972), Biochim. Biophys. Acta 289, 352-358.

10. Goedde, H. W., Münsch, H. \& Scholler, K. L. (1975), Anaesthesiol. (im Druck).

11. Whittaker, V. P. \& Wijesundera, S. (1951), Biochem. J. 52, 475-479.

12. Goedde, H. W., Doenicke, A. \& Altland, K. (1967), „Pscudocholinesterasen", 1. Aufl., S. 71, Springer Verlag, BerlinHeidelberg-New York.

13. Goedde, H. W., Held, K. \& Altland, K. (1968), Mol. Pharmacol. 4, 274-278.

14. Hobbiger, F. \& Peck, A. W. (1969), Brit. J. Pharmacol. 37, 258-271. erkennen, die ebenfalls für eine verlängerte Apnoe nach Succinylbischolin verantwortlich sein könnten. Derartige Untersuchungen, zu deren Durchführung der hier beschriebene Test entwickelt wurde, führen wir zurzeit in größerem Maßstab durch.

Andere Studien, die mit Hilfe der hier beschriebenen Methode in Angriff genommen wurden, befassen sich mit einer eventuellen Organspezifität der Cholinesterasen, über die bislang nur wenige Ergebnisse vorliegen (24-26).

\section{Danksagung}

Wir danken der Deutschen Forschungsgemeinschaft und der Alexander von Humboldt-Stiftung, Bonn-Bad Godesberg, für ihre Unterstützung.

15. Fishtal,A., Evans, R. T. \& Chapman, C. N. (1972), J. Clin. Pathol. 25, 422-426.

16. Hageboom, G. H. (1955), Methods of Enzymology I, Colowick, S. P. \& Kaplan, N. O., ed., p. 17 ff, Academic Press Inc., New York.

17. Rothschild, H. A., Cori, O. \& Barron, E. S. G. (1954), J. Biol. Chem. 208, 41-53.

18. Bockendahl, H. (1964), Hoppe Seyler's Z. Physiol. Chem. $336,172-175$.

19. Foldes, F. F., Foldes, V. M. \& Smith, J. C. (1962), 1. Europ. Kongress f. Anaesthesie, Wien 1962.

20. Thompson, J. C. \& Whittaker, M. (1966), Acta Genet. Basel, 16, 209-222.

21. Kalow, W. (1966), Anaesthesist 15, 13-18.

22. Goedde, H. W., Altland, K. \& Schloot, W. (1968), Ann. N. Y. Acad. Sci. $151,742-752$.

23. Whittaker, M. \& Vickers, M. D. (1970), Brit. J. Anaesthes. 42, 1016-1020.

24. Liddel, J., Newman, G. E. \& Brown, D. F. (1963), Nature (London) 198, 1090-1091.

25. Ramachandran, B. V. \& Ågren, G. (1963), Biochem. Pharmacol. 12, 981-988.

26. Smith, J. C. \& Foldes, F. F. (1963), Can. J. Biochem. Physiol. 41, 1713-1720.
Dr. D. P. Agarwal, Prof. Dr. H. W. Goedde Institut für Humangenetik der Universität Hamburg 2 Hamburg 54 Butenfeld 32 
